\title{
MicroBooNE cross-sections from an oscillation perspective
}

\author{
Xiao Luo*t \\ Yale University \\ E-mail: xiao.luo@yale.edu
}

The MicroBooNE experiment uses the Liquid Argon Time Projection Chamber technology to study the properties of neutrino oscillations. In parallel, an active program of measuring $v$-Ar cross-sections is making rapid progress, which is desirable because while demonstrating the capability of producing high level physics results, can also directly impact the precision of the oscillation analyses. This talk reviews several on-going cross-section analyses and their connections in reaching MicroBooNE's oscillation goals.

The 19th International Workshop on Neutrinos from Accelerators-NUFACT2017

25-30 September, 2017

Uppsala University, Uppsala, Sweden

${ }^{*}$ Speaker.

${ }^{\dagger}$ On behalf of MicroBooNE collaboration. 


\section{Introduction}

The MicroBooNE detector is located at the near surface on Fermilab's Booster Neutrino Beamline (BNB) about $470 \mathrm{~m}$ away from the target. The average neutrino energy of the BNB flux is 700 $\mathrm{MeV}$ and the flux primarily consists of muon neutrinos with about $0.5 \%$ electron neutrino contamination [1]. MicroBooNE started operations in October 2015 and has collected $6.5 \times 10^{20}$ POT of neutrino data up to date.

MicroBooNE's primary oscillation goal is to determine the nature of the excess of $v_{e}$-like events observed in the low energy region by the MiniBooNE experiment. According to MiniBooNE's latest published results, $2.8 \sigma$ and $3.4 \sigma$ excess have been observed in anti- $v_{e}$ and $v_{e}$ appearance channels. Since a Cherenkov detector is not able to distinguish electrons from photons, a significant background of MiniBooNE $v_{e}$ like events are mis-identified $\pi^{0} \mathrm{~s}$ and $\gamma_{\mathrm{s}}$. This makes it difficult to reach a clear interpretation of the nature of these excess events. Sharing the same beamline and neutrino beam flux with MiniBooNE, MicroBooNE uses a Liquid Argon Time Projection Chamber (LArTPC), which can trace the passages of the particles within mm resolution, to separate electrons and photons, therefore allowing to unambiguously determine the nature of low energy excess events.

Recent progress in neutrino physics has shifted the field to precision measurement of neutrino oscillation. As part of this world effort, MicroBooNE's mission on oscillation physics has expanded beyond the MiniBooNE low energy excess, in particular, pioneering the search for $\Delta m^{2} \sim 1 \mathrm{eV}^{2}$ sterile neutrinos with $5 \sigma$ sensitivity as one of the three experiments of Fermilab Short Baseline Neutrino (SBN) program, and providing data driven constrains on $v-A r$ cross-section uncertainties for the future accelerator based long baseline neutrino experiment - DUNE.

The rest of this proceeding will provide dedicated discussions about several on-going analyses in the MicroBooNE cross-section program and their direct impact on achieving these three oscillation goals.

\section{2. $v$ - Ar Cross-sections and Impact on Oscillation physics}

A typical neutrino oscillation analysis ( $v_{\mu}$ disappearance or $v_{e}$ appearance) can be simplified as three steps: (1) Signal selection (2) $v$ energy reconstruction (3) Estimation of systematic uncertainty. Cross-section analyses share the same procedure, hence can provide valuable experience to each of the above steps. Accurate $v-A r$ cross-section results from MicroBooNE are especially necessary for the following four reasons:

- Build confidence on the capability of producing high level physics results from large scale LArTPC detectors.

- Enable comparisons with other neutrino scattering data with different target materials and provide inputs to tune neutrino generators and theoretical models.

- Direct measurement of $v_{\mu}$-Ar cross-sections can be scaled for the $v_{e}-\mathrm{Ar}$ cross-sections, providing data-driven rate constrain for the $v_{e}$ appearance analysis. 
- Provide constrains on the systematic uncertainty introduced from the nuclear effects. Oscillation parameters are extracted from true neutrino energy distribution. Unfolding from reconstructed neutrino energy to true neutrino energy unavoidably relies on simulation. Depending on the different topological content of the signal selection, the unfolding matrices are different. Systematic uncertainty from nuclear effects enters at this step.

\section{1 $v_{\mu}$ Charged current $\left(v_{\mu} C C\right)$ inclusive cross-section}

The $v_{\mu} C C$ inclusive cross-section is the first measurement chosen in the MicroBooNE cross-section program. The only topological requirement to select $v_{\mu} \mathrm{CC}$ inclusive events is a muon track produced from the neutrino vertex. The muon kinematics is insensitive to final state interaction (FSI). This channel can be used as a standard candle to compare with other neutrino experiments, particularly the only existing $v-A r$ cross-section result from the ArgoNeuT experiment.

The performance of preliminary selection in MicroBooNE showed 30\% efficiency and 65\% of purity and good agreement between data and simulation in terms of the shape of muon kinematics distribution [2]. The current efforts to improve the selection efficiency are focused on better cosmic background rejection with scintillation light information and better muon particle identification using calorimetric information. The goal of the improved $v_{\mu} C C$ selection is to produce a double differential cross-section in the phase space of muon momentum and direction. Near future results are expected.

From a neutrino oscillation point of view, $v_{\mu} C C$ events are not only the signal channel of the $v_{\mu}$ disappearance analysis, but also provide the rate (flux $\times$ cross-section) constrain of the beam intrinsic $v_{e}$ events, which is the dominant, irreducible background of the $v_{e}$ appearance analysis. MicroBooNE's low energy excess analysis is currently pursuing both exclusive and inclusive $v_{e}$ selections, and the constrains of the inclusive $v_{e}$ rate will come from the improved $v_{\mu} C C$ inclusive cross-section analysis.

\section{2 $\mathrm{CC} \pi^{0}$ cross-section}

All three goals of the MicroBooNE oscillation analysis (low energy excess, sterile neutrino search, and $v_{e}$ appearance study for DUNE), involve reconstruction and selection of $v_{e} C C$ events. The signature of these events in liquid argon is one EM shower (induced by $\mathrm{e}^{-}$) attached to the neutrino interaction vertex. The small $v_{e}$ contamination in the BNB dominated by $v_{\mu}$ s forbids the effective development for the shower reconstruction. Fortunately, the $\pi^{0}$ s produced from the $v_{\mu} C C$ events can provide the large sample to study the EM photon showers from the $\pi^{0}$ decay, and shed the first light on the potential of e/ $\gamma$ separation in LArTPC.

The signature of $\mathrm{CC} \pi^{0}$ events is a muon track and two showers originating from a common vertex. The shower start points often have gaps from the neutrino vertex (see the right graph in figure 1 as an example of a $\mathrm{CC} \pi^{0}$ event candidate). Besides the common challenge of finding such neutrino interaction in large cosmic background, accurate $\pi^{0}$ reconstruction is also difficult due to the stochastic nature of the EM shower. Accurate 2D clustering and 3D shower reconstruction are being developed for the $\mathrm{CC} \pi^{0}$ cross-section analysis.

The $\mathrm{CC} \pi 0$ cross-section analysis also provides the photon sample for the important e/ $\gamma$ separation study. Two well known handles can be used to separate photons from electrons: $\mathrm{dE} / \mathrm{dx}$ at the 

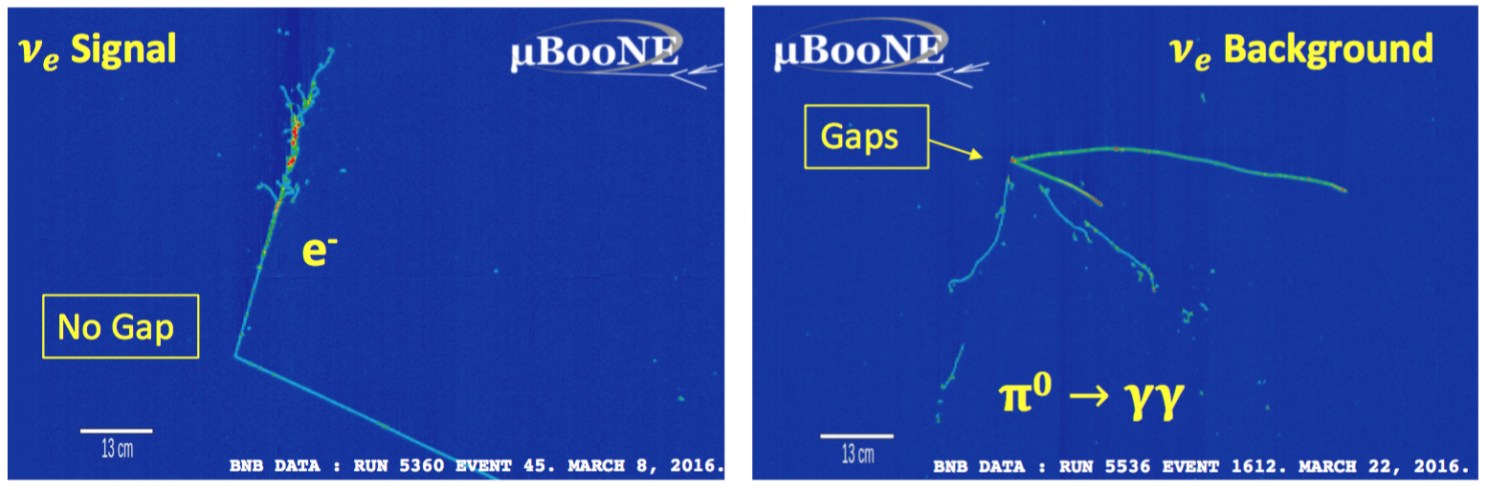

Figure 1: MicroBooNE Event display examples: candidate $v_{e} C C$ event (left), candidate $v_{\mu} C C \pi^{0}$ event (right).

shower start, and existence of gaps between the neutrino vertex and shower start. Photons are visible in the TPC only through two processes: Compton scattering with an outgoing electron, and pair production to electron and position. In principle, the $\mathrm{dE} / \mathrm{dx}$ of the shower start from Compton scattering process corresponds to 1 Minimally Ionized Particle (MIP), and pair production corresponds to 2 MIPs. Additionally, how far the photon travels before Compton scattering or pair productions sets the distance of the gaps between shower start and neutrino vertex ${ }^{1}$. As for electrons, $\mathrm{dE} / \mathrm{dx}$ corresponds to $1 \mathrm{MIP}$ at the shower start, and no gap between shower start and neutrino vertex. It is worth emphasizing both $\mathrm{dE} / \mathrm{dx}$ and gap handles should be used simultaneously in order to achieve strong e/ $\gamma$ separation.

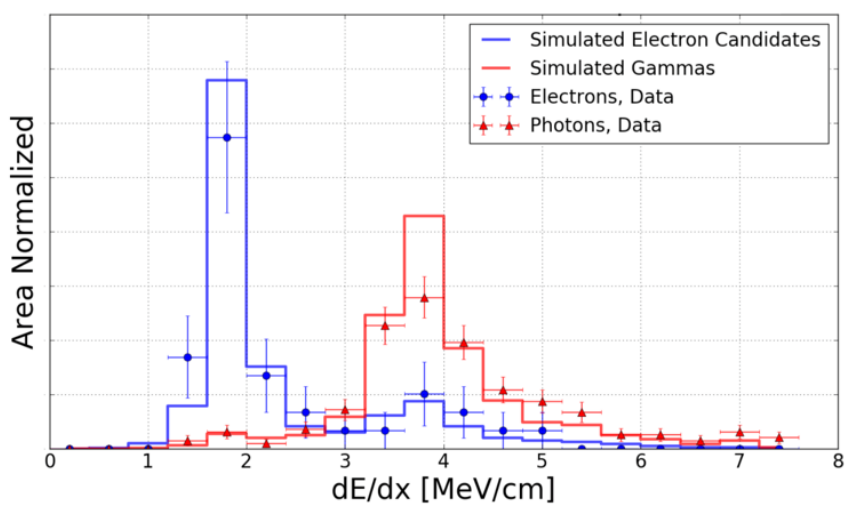

Figure 2: The dE/dx distribution for electrons and photons from ArgoNeuT.[3]

Figure 2 shows an example of e/ $\gamma$ separation using the medium value of $\mathrm{dE} / \mathrm{dx}$ distributions from ArgoNeuT data [3]. With the distinct 1MIP peak from electron data and 2MIP peak from the photon data, the paper quoted a $\sim 20 \%$ photon contamination in the observed electron candidate data sample and $\sim 7 \%$ photon contamination if place the $\mathrm{dE} / \mathrm{dx}$ cut at $2.9 \mathrm{MeV} / \mathrm{cm}$. Note that the amount of photon contamination heavily depends on the shower energy and technique of extracting

\footnotetext{
${ }^{1}$ The distance of $\pi^{0}$ travels before decay is negligible.
} 
$\mathrm{dE} / \mathrm{dx}$. For instance, higher energy photons are more likely to pair produce than Compton scatter, therefore less fraction of these photons have $\mathrm{dE} / \mathrm{dx}$ located at $1 \mathrm{MIP}$ region. Neutrinos in ArgoNeuT are from Fermilab's NuMI beam, which is higher energy than the BNB neutrinos in MicroBooNE. This means the photon contamination (from irreducible Compton scattering background) in MicroBooNE could be potentially higher than ArgoNeuT, assuming the same reconstruction and $\mathrm{dE} / \mathrm{dx}$ extraction technique. Rapid progress has been made on developing e/ $\gamma$ separation and shower reconstruction, and we expect to have the first $\mathrm{CC} \pi^{0}$ cross-section result in the near future.

\subsection{Charged current tack multiplicity and proton multiplicity}

Due to the complex nuclear effects, experiments normally categorize events based on their topology. MicroBooNE is currently performing measurements on track multiplicity and proton multiplicity. These measurements provide standard language to compare with other experiments, as well as tuning of the model parameters in the generators.

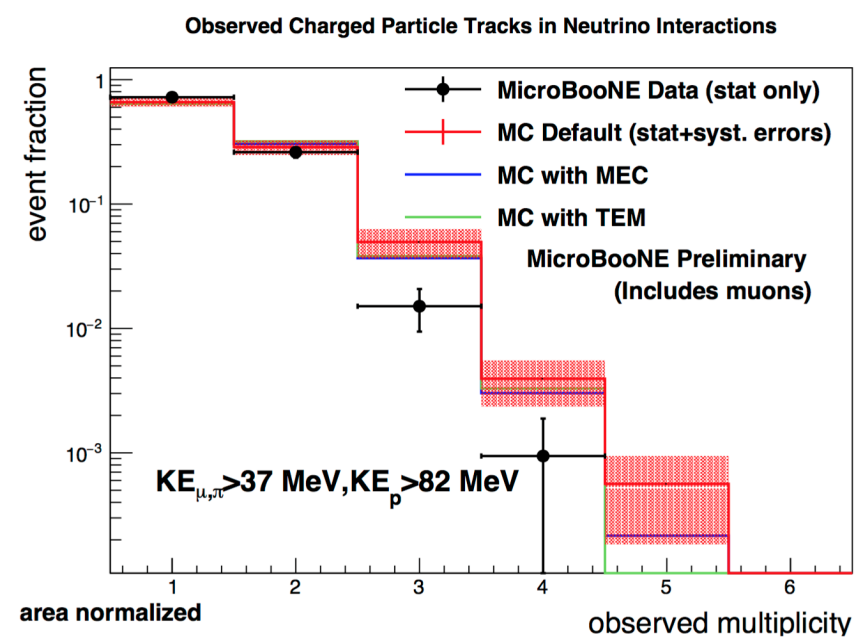

Figure 3: Area normalized, cosmic background subtracted track multiplicity distribution from MicroBooNE data overlaid with three GENIE predictions in log y scale. [4]

The track multiplicity analysis directly counts the number of reconstructed tracks originated from the same vertex. Multiple Coulomb Scattering and calorimetry are used to determine the track direction to minimize the cosmic background. In this analysis, relatively high energy thresholds are used to select the outgoing tracks ( $37 \mathrm{MeV}$ for $\mu$ and $\pi$, and $82 \mathrm{MeV}$ for protons). The preliminary result of the track multiplicity measurement is shown in figure 3. Overall a good agreement is observed between data and MC with less high track multiplicity events $(\mathrm{N}>=3)$ in data. There is not enough sensitivity for the data to show preference of different GEINE settings. Details of the event reconstruction, selection and data-driven techniques to estimate the cosmic background contamination can be found in [4]. The analysis is currently being completed with improved statistics and reduced energy thresholds.

The cross-section measurements of exclusive channels of $v_{\mu} C C 0 \pi$ (with different number of outgoing protons) are being pursued in MicroBooNE. The performance of these analyses are 
driven by the track reconstruction efficiency and proton identification. Current track reconstruction efficiencies are $95.8 \%$ for muons and $87.3 \%$ for protons [5], and single proton events with energy as low as $40 \mathrm{MeV}$ can be reconstructed and identified [6]. From a nuclear physics point of view, proton multiplicity measurement is particularly sensitive to different models that describe nuclear effects, such as nucleon correlation, final state interaction, etc. Different nuclear effect models results in different shape of $C C O \pi$ cross-section as a function neutrino energy. In practice, the oscillation analysis relies on a set of models in the generator to unfold the reconstructed neutrino energy. The correctness of the nuclear effects modeling determine the precision of the true neutrino energy. The constrain of these nuclear effects can be obtained from experimental data. For example, the $1 \mu 2 \mathrm{p}$ events can be used to tune the $2 \mathrm{p} 2 \mathrm{~h}$ model in the generator.

The ArgoNeuT experiment once again pioneered the proton multiplicity measurement. They have measured the $\mathrm{CC} 0 \pi$ cross-section for both neutrino and anti-neutrino data and GENIE prediction are 64\% (for neutrino) and 22\% (for anti-neutrino) higher than data. Larger difference between $\mathrm{MC}$ and data also appears at the higher proton multiplicity channels [7]. This result hints that more accurate FSI models are needed in the generators and more experimental data on argon is needed to cross-check ArgoNeuT result and constrain the nuclear effects in the generators. It is worth noting that the observed difference of the data/MC discrepancy for neutrino and anti-neutrino result is particularly relevant to the $\delta_{C P}$ measurement in the long-baseline oscillation analysis. Conceptually in order to reach the high sensitivity for the measurement of $\delta_{C P}$, generators need to include the nuclear effects that can describe both neutrino and anti-neutrino data simultaneously, to prevent any additional uncertain $v / \bar{v}$ asymmetry at unfolding stage. In reality, the difference of the nuclear effects will enter the systematic uncertainty to $\delta_{C P}$.

\section{Conclusion}

MicroBooNE studies neutrino oscillation with the goals of understanding the MiniBooNE low energy excess, searching for $\Delta m^{2} \sim 1 \mathrm{MeV}^{2}$ sterile neutrinos, and providing $v-\mathrm{Ar}$ cross-section constrain for Dune. I have demonstrated the impact on achieving these oscillation goals from several active cross-section analyses $\left(v_{\mu} \mathrm{CC}, \mathrm{CC} \pi^{0}\right.$, track and proton multiplicity measurements) in MicroBooNE. Physics results are expected soon to inform the broader neutrino community with $v$-Ar data obtained by large scale LArTPC.

\section{References}

[1] MicroBooNE collaboration, "Design and construction of the MicroBooNE detector", JINST 12, P02017 (2017)

[2] MICROBOONE-NOTE-1010-PUB, "Selection and kinematic properties of $v_{\mu}$ charged-current inclusive events in $5 \times 10^{19}$ POT of MicroBooNE data"

[3] ArgoNeuT Collaboration, "First Observation of Low Energy Electron Neutrinos in a Liquid Argon Time Projection Chamber", Phys. Rev. D95, 072005 (2017)

[4] MICROBOONE-NOTE-1024-PUB, "Measurement of Reconstructed Charged Particle Multiplicities of Neutrino Interactions in MicroBooNE"

[5] MicroBooNE collaboration, "The Pandora multi-algorithm approach to automated pattern recognition of cosmic-ray muon and neutrino events in the MicroBooNE detector", arXiv:1708.03135 
[6] MICROBOONE-NOTE-1025-PUB, "Proton Track Identification in MicroBooNE simulation for Neutral Current Elastic Events"

[7] Ornella Palamara, "CC 0 pion: ArgoNeuT results \& future prospects in LAr detector", presentation in NUINT 2015. 